\title{
Exchange energy enhanced $g$-factors obtained from Landau fan diagrams at low magnetic fields
}

\author{
S. Nomura* \\ Division of Physics, University of Tsukuba, Tennodai, Tsukuba, 305-8571, Japan \\ H. Tamura, M. Yamaguchi, and T. Akazaki ${ }^{\dagger}$ \\ NTT Basic Research Laboratories, NTT Corporation, Morinosato-Wakamiya, Atsugi, 243-0198, Japan \\ Y. Hirayama \\ Department of Physics, Faculty of Science, Tohoku University, Aoba, Aobaku, Sendai, 980-8578, Japan \\ WPI-AIMR, Tohoku University, Katahira, Aobaku, Sendai, 980-0812, Japan and ERATO-JST, \\ Katahira, Aobaku, Sendai, 980-0812, Japan
}

(Received 28 November 2012; published 25 February 2013)

\begin{abstract}
We report on measurement of the electron-hole effective $g$-factors $\left(g_{\text {eff }}^{*}\right)$ depending on electron filling factors $v$ from magnetophotoluminescence spectroscopy at low magnetic fields $B<1 \mathrm{~T}$ in low electron density regime in a GaAs $/ \mathrm{Al}_{0.33} \mathrm{Ga}_{0.67} \mathrm{As}$-gated quantum well. Enhancement of $g_{\text {eff }}^{*}$ at odd $v$ is observed. The oscillatory behavior of $g_{\text {eff }}^{*}$ is compared with results of a theory that takes into account the lowest-order exchange interaction of the screened Coulomb interaction. Good agreement of the observed $g_{\text {eff }}^{*}$ with theoretical results is obtained except $v$ at around 3. The enhancement of $g_{\text {eff }}^{*}$ at even $v$ with decrease in electron density has not been observed.
\end{abstract}

DOI: 10.1103/PhysRevB.87.085318 PACS number(s): 78.67.De, 73.43.-f, 71.70.Di, 71.70.Gm

\section{INTRODUCTION}

The recombination of electrons in two-dimensional electron system (2DES) in Landau quantized orbitals with valence band holes has been recognized as one of the ideal probes for the study of electron-electron and electron-hole interactions. ${ }^{1}$ The photoluminescence (PL) energy and intensity show oscillation with the electron filling factor $v{ }^{2,3}$ This is understood to be due to oscillations of quasiparticle self-energies of electrons and holes. The majority of the existing studies of the oscillations of the PL with $v$ was carried out at high magnetic field $B$ of several $\mathrm{T}$ to $\approx 20 \mathrm{~T} .{ }^{4}$ In high electron density, an optically created hole only weakly perturbs the 2DES. In lower magnetic field and in lower electron density, the ratios of the electron-electron and electron-hole energies to the cyclotron energy increase, and phenomena due to the electron-electron and electron-hole interactions are expected to be more prominent. In fact, it has been found in GaAs/AlGaAs heterostructures and in Si inversion layers that the electron effective mass $\left(m_{e}^{*}\right)$ and spin susceptibility of electrons $\chi=m_{e}^{*} g^{*}$ increase with a decrease in electron density, ${ }^{5-8}$ where $g^{*}$ is the electron effective $g$ factor.

The increase in effective masses of the electron and the optically created hole was observed by photoluminescence (PL) spectroscopy in a GaAs/AlGaAs back-gated quantum well (QW), ${ }^{9}$ demonstrating that the PL spectroscopy is useful for studying the low-density electron systems. The splitting energy $\Delta E=E\left(\sigma^{+}\right)-E\left(\sigma^{-}\right)$is, in principle, obtained by direct measurement of the PL transition energies for $\sigma^{+}$ and $\sigma^{-}$circular polarizations, where the photon angular momentum $+1(-1)$ is assigned for $\sigma^{+}\left(\sigma^{-}\right) .{ }^{10}$ While this is a standard method for determination of the effective $g$-factor of magnetoexcitons, ${ }^{11}$ the measurement of the effective $g$-factor of 2DES by the splitting energy is relatively few. ${ }^{12,22}$ Goldberg et al. observed no enhancement of $g$-factor determined from the optical transition energies at $v=1,{ }^{12}$ while the exchange enhanced $g$-factors were observed by inelastic light scattering at $v=1$ from long-wavelength spin-flip excitations, ${ }^{7,13}$ by avoiding the influence of the disorder potential. The disorder potential is known to collapse the exchange enhancement of the electron $g$-factor. ${ }^{14,15}$ A clean electron system is especially important for investigation of the electron-hole system in the low electron density regime, where the excitonic correlation compete with the 2DES correlation of the electron fluids. However, the investigation of the PL in this regime is few because of the availability of high-quality samples in low density. Gated undoped $\mathrm{GaAs} / \mathrm{Al}_{x} \mathrm{Ga}_{1-x}$ As QWs are suitable for such investigations because of low disorder in the low electron density regime due to the lack of a donor layer and the ability to tune the electron density from nominally zero to the order of $1 \times 10^{11} \mathrm{~cm}^{-2}$ by gate voltage. .,16,17 $^{-17}$

In this paper, we report on systematic PL measurements of a gated undoped $\mathrm{GaAs} / \mathrm{Al}_{x} \mathrm{Ga}_{1-x} \mathrm{As} \mathrm{QW}$ sample in low electron density below $1 \times 10^{11} \mathrm{~cm}^{-2}$ in low perpendicular magnetic field $B \leqslant 1 \mathrm{~T}$, where the spin-split energy of a valence band hole is linear in $B$ in good approximation. ${ }^{11}$ The Landau fan diagrams are obtained from the circular polarization resolved PL peaks. The electron-hole effective $g$-factors as a function of the electron filling factor $v$ are directly derived from the energy separation of the circular polarization resolved PL peaks due to recombination of up- and down-spin electrons with a hole. We investigate the roles of the electron-electron and electron-hole interaction in the low electron density regime.

\section{EXPERIMENTAL}

The sample layer consists of $n$-GaAs, superlattice barrier, 20-nm $\mathrm{Al}_{0.33} \mathrm{Ga}_{0.67}$ As layer, 50-nm GaAs well, and 400-nm $\mathrm{Al}_{0.33} \mathrm{Ga}_{0.67} \mathrm{As}$ layer. The sample showed peak mobility of $3 \times 10^{6} \mathrm{~cm}^{2} / \mathrm{Vs}$. The back-gate bias voltage was applied between the surface side annealed contacts and the back contact. The linearly polarized laser light at $800 \mathrm{~nm}$ was irradiated on the sample in a dilution refrigerator using a polarization maintaining single-mode optical fiber at the 
incident power of $0.8 \mu \mathrm{W}$. The circular polarization resolved PL from a $0.5 \times 0.5-\mathrm{mm}^{2}$ square mesa structure was measured at base temperature of $20 \mathrm{mK}$ using a wave plate and a polarizer in the mixing chamber, an optical fiber with a $400-\mu \mathrm{m}$ core diameter, and a Spex 1404 double monochromator equipped with a liquid-nitrogen-cooled charge-coupled device detector.

\section{THEORY}

The electron $g$-factor in GaAs QW is calculated using the method by Ando and Uemura. ${ }^{18}$ Effects of the level broadening of each Landau level are treated by the self-consistent Born approximation (SCBA), and the self-energies of electrons are calculated by including the lowest-order exchange interaction of the screened Coulomb interaction. We assume a twodimensional electron gas and the finite width of the QW is not taken into account. The correlation energy of the electrons and the electron-hole Coulomb interaction are not taken into account. The self-energy for the LLN, where LLN stands for the $N$ th Landau levels, is given by the expression ${ }^{18,19}$

$$
\Sigma_{N \sigma}=-\sum_{q, N^{\prime}} \frac{V(q)}{\varepsilon(q)} J_{N, N^{\prime}}(q)^{2} n_{N^{\prime}, \sigma},
$$

where $V(q)=2 \pi e^{2} / \kappa q, \varepsilon(q)$ is the dielectric function, $J_{N, N^{\prime}}(q)$ is the matrix element between the Landau levels $N$ and $N^{\prime}$, and $n_{N \sigma}$ is the occupation number for the Landau level $N$ with spin $\sigma$. The $g$-factor for $\operatorname{LL} N g_{N}^{*}$ is expressed by

$$
\begin{aligned}
g_{N}^{*} \mu_{B} H & =g_{0} \mu_{B} H+\Sigma_{N \downarrow}-\Sigma_{N \uparrow} \\
& =g_{0} \mu_{B} H+\sum_{q, N^{\prime}} \frac{V(q)}{\varepsilon(q)} J_{N, N^{\prime}}(q)^{2}\left(n_{N^{\prime} \uparrow}-n_{N^{\prime} \downarrow}\right) .
\end{aligned}
$$

This expression for the $g$-factor for LL $N$ implicitly depends on the Fermi energy $E_{F}$ or the filling factor $\nu$. The $g$-factors for the lower (=completely filled) Landau level $\left(N^{\prime}<N\right)$ become $\left|g^{*}\right|>\left|g_{0}\right|$ when the Fermi energy lies in the $N$ th Landau level.

The level broadening depends on the magnetic field in the SCBA and is given by the expression

$$
\Gamma(B)=\left(\frac{2}{\pi} \hbar \omega_{c} \frac{\hbar}{\tau}\right)^{1 / 2},
$$

where $\omega_{c}$ is the cyclotron frequency for the magnetic field $B$ and $\tau$ is the scattering time under zero magnetic field. The level broadening relative to the Landau level separation $\hbar \omega_{c}$ is given by $\Gamma(B) / \hbar \omega_{c} \propto 1 / \sqrt{B}$, which means that the broadening becomes relatively narrower to $\hbar \omega_{c}$ as the magnetic field $B$ increases. In our calculation, the value of $\Gamma$ under $B$ is determined from $\Gamma_{0}=\Gamma(B=1)$; i.e., $\Gamma(B)=\sqrt{B} \Gamma_{0}$. We assume that the valence band hole $g$-factor $\left(g_{\mathrm{h}}\right)$ is electrondensity-independent. Then the electron-hole-effective $g$-factor is given by $g_{\mathrm{eff}}^{*}=g_{N}^{*}+g_{\mathrm{h}}$.

\section{RESULTS AND DISCUSSIONS}

Figures 1(a) and 1(b) show the PL spectra from a 2DES in perpendicular magnetic field $B \leqslant 1 \mathrm{~T}$ at the electron densities $n_{s}=5.0 \times 10^{10}$ and $1.0 \times 10^{11} \mathrm{~cm}^{-2}$ at a temperature $T=$ $20 \mathrm{mK}$. The magnetic field was swept by fixing the orientation of a wave plate and a polarizer. The PL in positive and negative $B$ corresponds to the $\sigma^{-}$and $\sigma^{+}$circular polarizations, respec- tively. The PL in $\sigma^{-}\left(\sigma^{+}\right)$circular polarization corresponds to the recombination of a majority (minority) spin electron with a valence band hole. Plotting the peak energies of the PL as functions of $B$ derives Landau fan diagrams as shown in Figs. 1(c) and 1(d). The PL peaks due to the transition between $N_{e}$ and $N_{h}=0$ are primarily observed, where $N_{e}$ and $N_{h}$ are the Landau indices for the electron and the hole, respectively. The lowest PL peak in $\sigma^{+}$is the recombination of spin $\downarrow$ electron and $|-3 / 2, n=-1\rangle_{h}$ hole, and the lowest PL peak in $\sigma^{-}$is the recombination of spin $\uparrow$ electron and $|3 / 2, n=2\rangle_{h}$ hole in the cylindrical symmetry approximation, where $n$ is the angular momentum. ${ }^{10,12,20}$ Both $|-3 / 2, n=-1\rangle_{h}$ and $|3 / 2, n=2\rangle_{h}$ states are primarily heavy-hole character at low magnetic fields. ${ }^{10,21}$ The off-diagonal $\left(N_{e} \neq N_{h}\right)$ transitions are induced by the mixing of the Landau-levels and by the impurity scattering. The diagonal $\left(N_{e}=N_{h}\right)$ transitions are also observed in LL1 in some cases at $0.48 \leqslant B \leqslant 0.60 \mathrm{~T}$ in Fig. 1(c) and at $0.86 \leqslant B \leqslant 1.00 \mathrm{~T}$ in Fig. 1(d). The full width at half maximum of PL peaks is $0.3-0.6 \mathrm{meV}$. The minimum linewidth is limited by the energy distribution of holes, ${ }^{22}$ which corresponds to the hole temperature of about $3 \mathrm{~K}$. The hole distribution does not reach thermodynamical equilibrium due to short recombination lifetime. ${ }^{23}$

The oscillations of the PL peak energies and the differences in the PL energies for $\sigma^{+}$and that for $\sigma^{-}$are observed. In the Landau levels LL0 and LL1, $E\left(\sigma^{+}\right)>E\left(\sigma^{-}\right)$holds, indicating that the electron-hole effective $g$-factor $g_{\text {eff }}^{*}$ is negative in agreement with the literature. ${ }^{4} \mathrm{~A}$ reversal of the PL peak energies in LLN is observed for $N \geqslant 2$, where $E\left(\sigma^{+}\right)$ is lower than $E\left(\sigma^{-}\right)$. The electron $g$-factor is negative and the PL peak energies in this case is explained by the transition matrix element of the electron and the light-hole component of the hole $\mathrm{e}^{10}$ mixed by the electron-hole Coulomb interaction. Figures 1(e) and 1(f) show that the PL intensity in $\sigma^{-}$ polarization $\left(I_{\sigma^{-}}\right)$is larger than that in $\sigma^{+}$polarization $\left(I_{\sigma^{+}}\right)$ for $v \geqslant 4$, except a few cases, indicating that the populations of the up-spin electrons are larger than the populations of the down-spin electrons and, thus, $g_{\text {eff }}^{*}<0$ holds in accordance with the previous argument. There are, however, a few cases where the PL intensity in $\sigma^{+}$polarization is larger than that in $\sigma^{-}$polarization. This effect is particularly significant at $v<4$ and in smaller $n_{s}$. As shown in Fig. 1(e), the intensity of LL0 PL peak in $\sigma^{+}$is remarkably larger than that in $\sigma^{-}$at $v=3$. This is associated with a spin-dependent Pauli blocking. In a partially filled Landau level at odd $v$, the mixings of wave functions with different angular momentum induced by the electron-hole Coulomb interaction are small for the up-spin electrons in the filled spin-split Landau level due to the Pauli blocking, while the mixings of wave functions are large for the down-spin electrons in the spin-split Landau level.

Figure 2(a) shows $g_{\text {eff }}^{*}$ obtained from the difference in the PL peak energies in $\sigma^{+}$and $\sigma^{-}$polarizations for LLO as given by $g_{\text {eff }}^{*}=\left[E\left(\sigma^{+}\right)-E\left(\sigma^{-}\right)\right] / \mu_{B}|B|$ by assuming $E_{\text {Zeeman }}=$ $\pm \frac{1}{2} g_{\text {eff }}^{*} \mu_{B} B$ for the electron density between $2.9 \times 10^{10}$ and $1.0 \times 10^{11} \mathrm{~cm}^{-2}$. In the following, we focus on LL0. The oscillations of $g_{\text {eff }}^{*}$ with $v$ are clearly seen with maxima of $\left|g_{\text {eff }}^{*}\right|$ at odd $v$ and minima at even $v$. The width of maxima of $\left|g_{\text {eff }}^{*}\right|$ at a fixed odd $v$ is smaller for larger $n_{s}$. The effective masses of the electron and the hole at $2.9 \times 10^{10} \mathrm{~cm}^{-2}$ are estimated to be $m_{e}^{*}=0.14 m_{0}$ and $m_{h}^{*}=0.39 m_{0}$ with the 

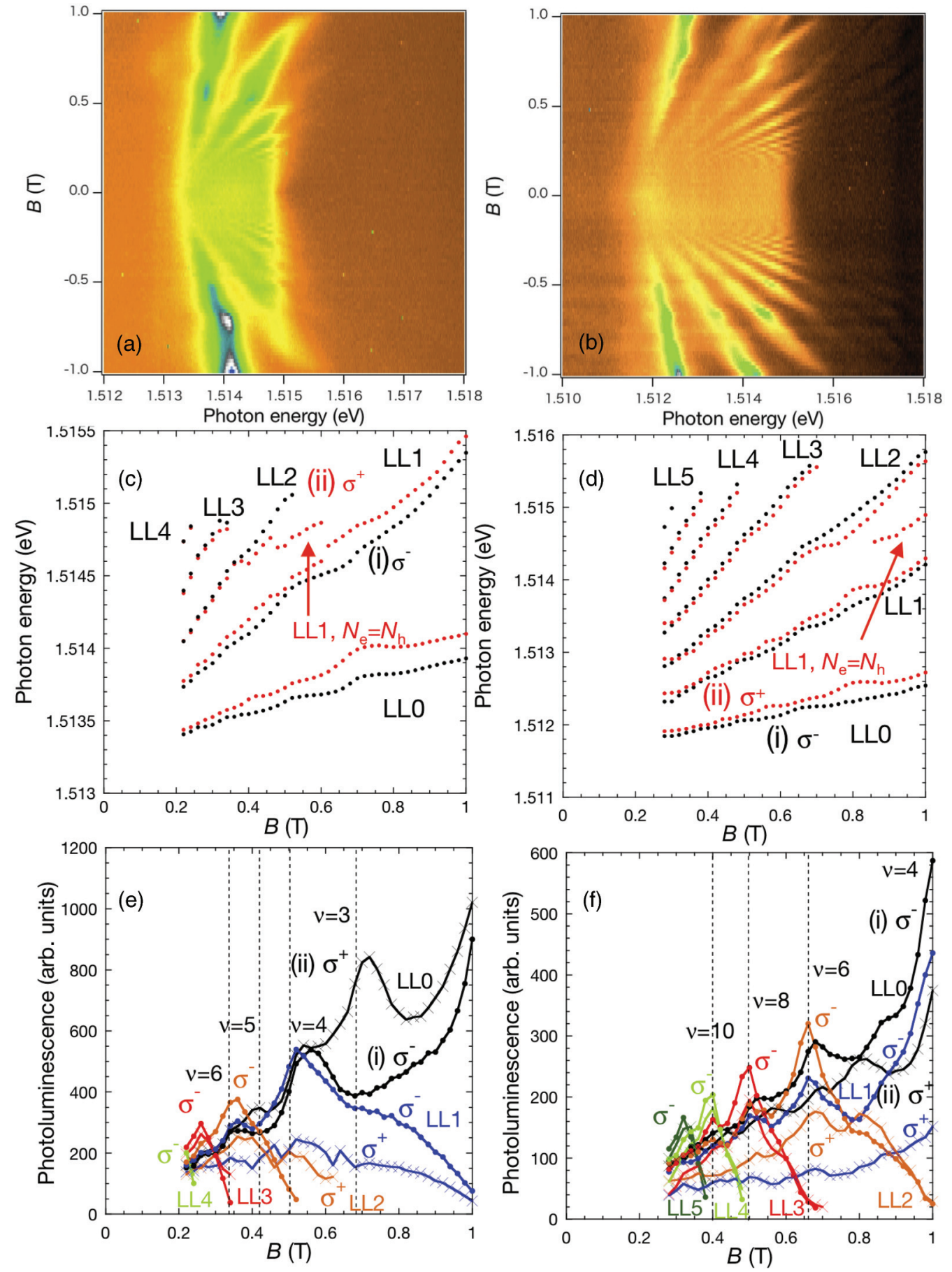

FIG. 1. (Color online) (a) Typical PL spectra in color-image plots for $n_{s}=5.0 \times 10^{10} \mathrm{~cm}^{-2}$ and (b) for $n_{s}=1.0 \times 10^{11} \mathrm{~cm}^{-2}$ at $T=20 \mathrm{mK}$. (c) Magnetic field dependence of PL peak energies for $n_{s}=5.0 \times 10^{10} \mathrm{~cm}^{-2}$ and (b) for $n_{s}=1.0 \times 10^{11} \mathrm{~cm}^{-2}$ in (i) $\sigma^{-}$(black) and (ii) $\sigma^{+}$(red) circular polarizations. (e) Magnetic field dependence of PL peak intensities for $n_{s}=5.0 \times 10^{10} \mathrm{~cm}^{-2}$ and (f) for $n_{s}=1.0 \times 10^{11} \mathrm{~cm}^{-2}$ in (i) $\sigma^{-}$(dots) and (ii) $\sigma^{+}$(crosses) circular polarizations, for LL0 (black), LL1 (blue), LL2 (orange), LL3 (red), LL4 (green), and LL5 (dark green).

method described in the literature ${ }^{9}$ by using the average of PL peak energies for $\sigma^{-}$and $\sigma^{+}$polarizations. This indicates that the hole is not localized in the range of the electron density shown in Fig. 2(a).

Figure 2(b) shows the calculated results of the $g$-factor for the transition energy from the lowest Landau level LL0, $g_{\text {eff }}^{*}=g_{N=0}^{*}+g_{\mathrm{h}}$, as functions of $\nu$, where we used parameters $\Gamma_{0}=0.16$ and $g_{\text {eff }}^{0}=g_{0}+g_{\mathrm{h}}=-2.0$, and the contribution of the valence band hole $g$-factor $\left(g_{\mathrm{h}}\right)$ is included in $g_{\text {eff }}^{0}$. The parameter $\Gamma_{0}$ was set to obtain good agreement with the measured values at odd $v$ as shown in Fig. 3, and $g_{\text {eff }}^{0}$ was set by using the experimentally obtained values of $g_{\text {eff }}^{*}$ at $v=10$ for LLO at $n_{s}=8.6 \times 10^{10}$ and at $1.0 \times 10^{11} \mathrm{~cm}^{-2}$.

The $v$ dependence of the calculated $g$-factors is plotted in Fig. 2(b) for fixed total electron density $n_{s}=v /\left(2 \pi l^{2}\right)=$ $(e / h) v B$. For example, $v B=1(\mathrm{~T})$ corresponds to the electron 

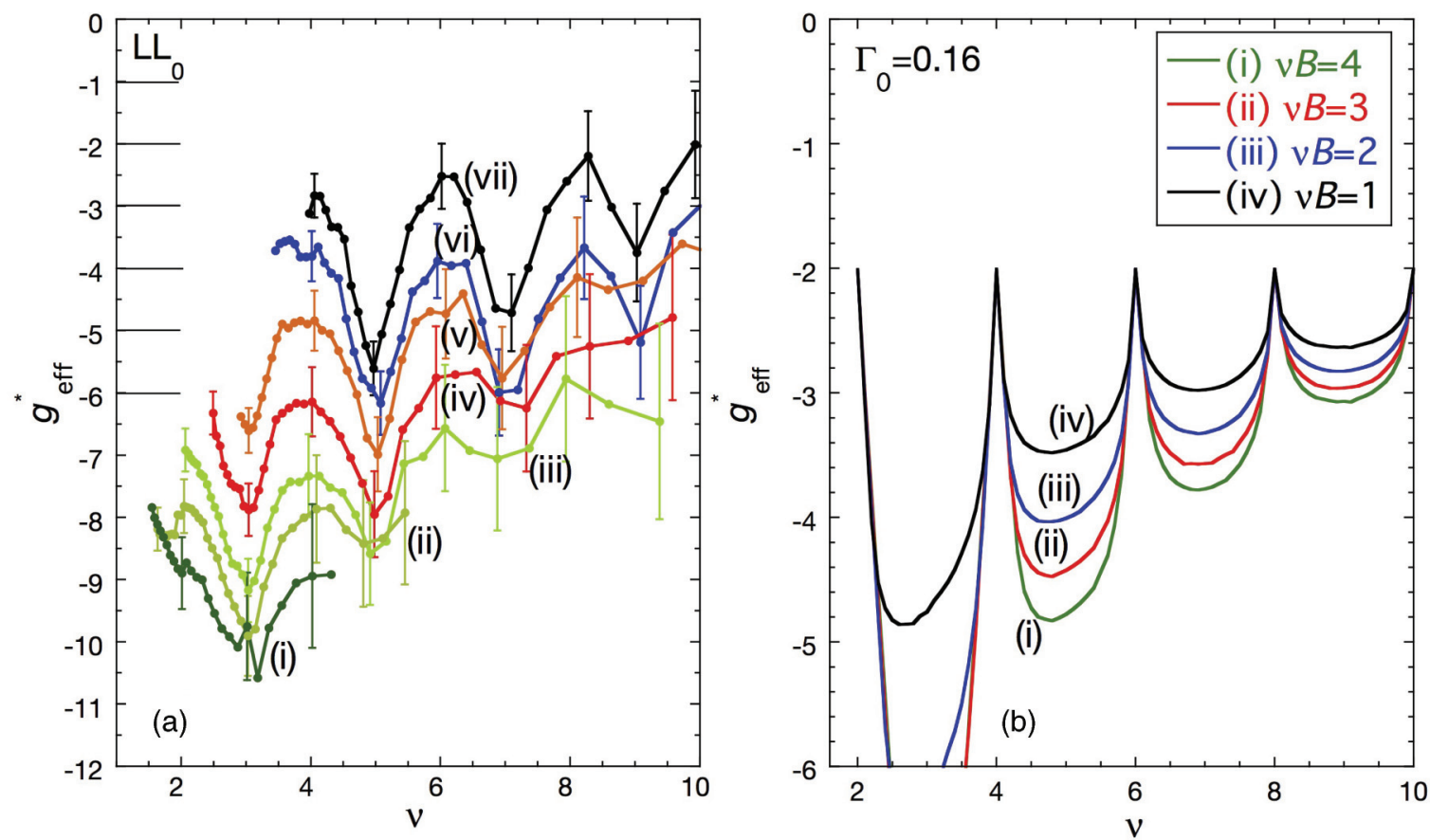

FIG. 2. (Color online) (a) Observed $v$-dependent $g_{\text {eff }}^{*}$ for $n_{s}=$ (i) $2.9 \times 10^{10}$, (ii) $4.0 \times 10^{10}$, (iii) $5.0 \times 10^{10}$, (iv) $6.0 \times 10^{10}$, (v) $7.1 \times 10^{10}$, (vi) $8.6 \times 10^{10}$, and (vii) $1.0 \times 10^{11} \mathrm{~cm}^{-2}$. The origin of the vertical axis is shifted. Error bars in $g_{\text {eff }}^{*}$ are shown for some representative cases. (b) Calculated $g$-factors using $\Gamma_{0}=0.16$ for $v B=$ (i) 4 , (ii) 3 , (iii) 2 , and (iv) 1 .

density of $n_{s}=(e / h) \times 1(\mathrm{~T})$. The calculated $\left|g_{\text {eff }}^{*}\right|$ has peaks around odd $v$, where the exchange interaction is large and drops down to $g_{\text {eff }}^{0}$ at even $v$, where there is no net spin. Larger $v$ gives smaller $g$-factor for smaller exchange interaction. The increase of $\left|g_{\text {eff }}^{*}\right|$ at even $v$ with decrease in $v$ in Fig. 2(a) may be explained by disorders that are not taken into account by Eq. (3); for example, long-range potential fluctuations. ${ }^{17,24}$

Figures 3(a)-3(d) show magnetic field dependence of the observed $g_{\text {eff }}^{*}$ at $v=3,5,7$, and 10. At a fixed $v$, larger magnetic field, i.e., larger $n_{s}$, gives larger $g_{\text {eff }}^{*}$. The calculated results are also shown in Figs. 3(a)-3(c). The $g$-factor gradually increases as $B$ since the relative level-broadening decreases as $1 / \sqrt{B}$, in agreement with the observations in Figs. 3(b) and 3(c). As the filling factors increase, the exchange interaction becomes smaller, resulting in smaller $g$-factors.

The observed $g_{\text {eff }}^{*}$ at $v=3$ shows saturation at $0.68 \mathrm{~T}$ as shown in Fig. 3(a), and $\left|g_{\text {eff }}^{*}\right|$ is smaller at $v=3$ than $\left|g_{\text {eff }}^{*}\right|$ at $v=5$ at $n_{s}=6.0$ and $7.1 \times 10^{10} \mathrm{~cm}^{-2}$. This saturation of $g_{\text {eff }}^{*}$ at $v=3$ cannot be explained by the calculated result as shown in Fig. 3(a) and was not observed in the activation energy measurements where the effective electron $|g|$-factors were obtained to be 5.3 and 2.5 at $v=3$ and 5 , respectively. ${ }^{25}$ The enhancement of $g$-factor up to 15 was observed at $v=3$ at $B \approx 6 \mathrm{~T}$ in $\mathrm{GaAs} / \mathrm{Al}_{x} \mathrm{Ga}_{1-x}$ As single heterojunction with a $\delta$ layer of $\mathrm{Be}$ acceptors located at a well-defined distance from the interfaces. ${ }^{22}$ It is tempting to associate the shrinking of the $\left|g_{\text {eff }}^{*}\right|$ at $v=3$ with the multiple low-energy spin excitations. ${ }^{26}$ However, the skyrmion at $v=3$ is fragile and it was found that Landau-level mixing tends to favor spinpolarized quasiparticles. ${ }^{27}$ It was indicated that the lowestlying charged excitations at $v=3$ was accompanied with a single spin flip at $B \geqslant 2 \mathrm{~T}^{28}$ Depolarization of the degree of circular polarization of the PL $P=\left(I_{\sigma^{-}}-I_{\sigma^{+}}\right) /\left(I_{\sigma^{-}}+I_{\sigma^{+}}\right)$ is expected at $v>3$ and at $v<3$ for the skyrmion ground state. The PL intensities at around $v=3$ in Fig. 1(e) are not consistent with formation of skyrmions. Minima of circular polarization $P$ in the LLO were previously observed at oddinteger filling factors 3,5 , and 7 in $\mathrm{GaAs} / \mathrm{Al}_{x} \mathrm{Ga}_{1-x} \mathrm{As}$ heterojunction samples, with a $\delta$ layer of Be acceptors at electron density of $n_{s}=1.7$ and $4.7 \times 10^{11} \mathrm{~cm}^{-2} .^{29}$ Their observation is in agreement with magnetic-field dependence of PL peak intensities as shown in Fig. 1(f) for $n_{s}=1.0 \times 10^{11} \mathrm{~cm}^{-2}$. In the lower electron density case of $n_{s}=5.0 \times 10^{10} \mathrm{~cm}^{-2}$, $P$ at $v=3$ further decreases to $P<0$ as shown in Fig. 1(e). The degree of circular polarization $P$ at around $v=3$ does not directly reflect the spin polarization of the electrons.

The saturation of $g_{\text {eff }}^{*}$ and the reduction of $P$ at $v=3$ is associated with the mixing of the Landau levels. The interLandau level coupling is significant at $1 \mathrm{~T}$ since the cyclotron energy $\hbar \omega_{c} \simeq 1.7 \mathrm{meV}$ is much less than the Coulomb energy $E_{0}=\sqrt{\pi / 2} e^{2} /\left(\kappa l_{B}\right) \simeq 5.5 \mathrm{meV}$, where $l_{B}$ is the magnetic length. As mentioned before, the PL intensity at around $v=3$ is larger in $\sigma^{+}$than in $\sigma^{-}$, indicating the excitonic enhancement of the oscillator strength in $\sigma^{+}$at around $v=3$. In the absence of the Coulomb interaction, spin-down LL0 is fully occupied and spin-down LL1 is empty at $v=3$. The excitonic enhancement of the oscillator strength in $\sigma^{+}$is induced by rearranging the occupations of the down-spin electrons in LLO and in empty LL1 in response to optically created hole in the valence band. ${ }^{30}$ Rearrangement of the occupations between the up-spin electrons in LLO and those in LL1 is small because both the spin-up LLO and LL1 levels are fully occupied at $v=3$ in 

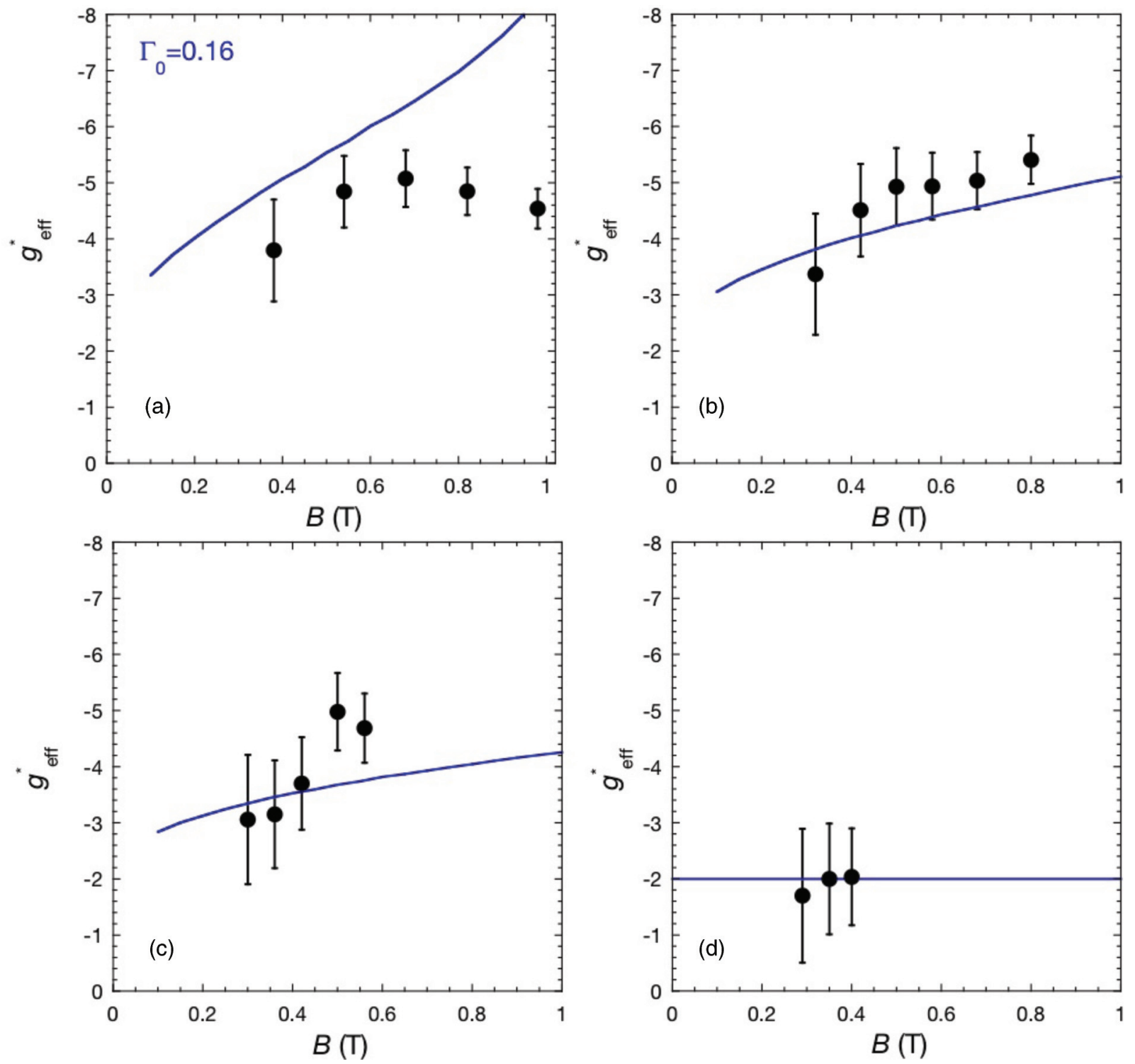

FIG. 3. (Color online) Magnetic field dependence of the observed $g$-factors $g_{\text {eff }}^{*}$ (solid circles) and calculated $g_{\text {eff }}^{*}$ using $\Gamma_{0}=0.16$ and $g_{\text {eff }}^{0}=-2.0$ (solid curves) at (a) $v=3$, (b) 5, (c) 7, and (d) $10 . g_{\text {eff }}^{*}=g_{\text {eff }}^{0}$ at $v=10$.

the absence of the Coulomb interaction. This excitonic effect causes larger reduction of the transition energy in $\sigma^{+}$than in $\sigma^{-}$, and hence the reduction of $\left|g_{\text {eff }}^{*}\right|$.

The observed $\left|g_{\text {eff }}^{*}\right|$ at even $v$ does not increase with decrease in $n_{s}$ in the range $n_{s} \geqslant 2.9 \times 10^{10} \mathrm{~cm}^{-2}$. This is consistent with the theory, including the lowest-order exchange interaction of the screened Coulomb interaction, and indicates that the electron correlations are not important at $n_{s} \geqslant 2.9 \times 10^{10} \mathrm{~cm}^{-2}$. Nevertheless, since $m_{e}^{*}$ increases with decrease in $n_{s},{ }^{9}$ spin susceptibility $\chi$ increases with decrease in electron density in the range $2.9 \times 10^{10} \leqslant n_{s} \leqslant 1.0 \times 10^{11} \mathrm{~cm}^{-2}$.

\section{CONCLUSIONS}

We have measured $g_{\text {eff }}^{*}$ at $B \leqslant 1 \mathrm{~T}$ for $3 \leqslant v \leqslant 10$ for the electron density between $2.9 \times 10^{10}$ and $1.0 \times 10^{11} \mathrm{~cm}^{-2}$. We have observed oscillatory behavior of $g_{\text {eff }}^{*}$ with $B$ with maxima of $\left|g_{\text {eff }}\right|$ of $\approx 5.6$ at odd $\nu$. The observed behaviors of $g_{\text {eff }}^{*}$ have been explained by a calculation taking into account the level broadening of each Landau level and the self-energies of electrons. It has been shown that $\left|g_{\text {eff }}^{*}\right|$ is larger for larger $B$ at a fixed odd $v$ because $\Gamma / \hbar \omega_{c}$ decreases with increase in $B$. We have found that the PL intensity in $\sigma^{+}$enhances and $\left|g_{\text {eff }}^{*}\right|$ decreases at $v=3$ because of the cancellation of the exchange-enhanced energy splitting by the excitonic effect. The enhancement of $\left|g_{\text {eff }}^{*}\right|$ at even $v$ with decrease in $n_{s}$ has not been observed.

\section{ACKNOWLEDGMENT}

This work was partly supported by Grant-in-Aid for Scientific Research "DYCE” No. 20104005. 
*nomura.shintaro.ge@u.tsukuba.ac.jp

†Present address: Kochi National College of Technology, Otsu Monobe Nankoku, 783-8508, Japan

${ }^{1}$ P. Hawrylak and M. Potemski, Phys. Rev. B 56, 12386 (1997).

${ }^{2}$ S. Katayama and T. Ando, Solid State Commun. 70, 97 (1989).

${ }^{3}$ T. Uenoyama and L. J. Sham, Phys. Rev. B 39, 11044 (1989).

${ }^{4}$ B. B. Goldberg, D. Heiman, M. Dahl, A. Pinczuk, L. Pfeiffer, and K. West, Phys. Rev. B 44, 4006 (1991).

${ }^{5}$ A. A. Shashkin, S. V. Kravchenko, V. T. Dolgopolov, and T. M. Klapwijk, Phys. Rev. Lett. 87, 086801 (2001).

${ }^{6}$ J. Zhu, H. L. Stormer, L. N. Pfeiffer, K. W. Baldwin, and K. W. West, Phys. Rev. Lett. 90, 056805 (2003).

${ }^{7}$ A. B. Van'kov, L. V. Kulik, I. V. Kukushkin, V. E. Kirpichev, S. Dickmann, V. M. Zhilin, J. H. Smet, K. von Klitzing, and W. Wegscheider, Phys. Rev. Lett. 97, 246801 (2006).

${ }^{8}$ B. Spivak, S. V. Kravchenko, S. A. Kivelson, and X. P. A. Gao, Rev. Mod. Phys. 82, 1743 (2010).

${ }^{9}$ S. Nomura, M. Yamaguchi, T. Akazaki, H. Tamura, T. Maruyama, S. Miyashita, and Y. Hirayama, Phys. Rev. B 76, 201306(R) (2007).

${ }^{10}$ S.-R. Eric Yang and L. J. Sham, Phys. Rev. Lett. 58, 2598 (1987).

${ }^{11}$ M. J. Snelling, E. Blackwood, C. J. McDonagh, R. T. Harley, and C. T. B. Foxon, Phys. Rev. B 45, 3922 (1992).

${ }^{12}$ B. B. Goldberg, D. Heiman, and A. Pinczuk, Phys. Rev. Lett. 63, 1102 (1989).

${ }^{13}$ A. Pinczuk, B. S. Dennis, D. Heiman, C. Kallin, L. Brey, C. Tejedor, S. Schmitt-Rink, L. N. Pfeiffer, and K. W. West, Phys. Rev. Lett. 68, 3623 (1992).

${ }^{14}$ M. M. Fogler and B. I. Shklovskii, Phys. Rev. B 52, 17366 (1995).
${ }^{15}$ W. Pan, K. W. Baldwin, K. W. West, L. N. Pfeiffer, and D. C. Tsui, Phys. Rev. B 84, 161307 (2011).

${ }^{16}$ Y. Hirayama, K. Muraki, and T. Saku, Appl. Phys. Lett. 72, 1745 (1998).

${ }^{17}$ M. Yamaguchi, S. Nomura, T. Maruyama, S. Miyashita, Y. Hirayama, H. Tamura, and T. Akazaki, Phys. Rev. Lett. 101, 207401 (2008).

${ }^{18}$ T. Ando and Y. Uemura, J. Phys. Soc. Jpn. 37, 1044 (1974)

${ }^{19}$ F. J. Ohkawa and Y. Uemura, J. Phys. Soc. Jpn. 43, 925 (1976).

${ }^{20}$ B. Goldberg, D. Heiman, A. Pinczuk, C. Tu, A. Gossard, and J. English, Surf. Sci. 196, 209 (1988).

${ }^{21}$ L. K. Castelano, D. F. Cesar, V. Lopez-Richard, G. E. Marques, O. D. D. Couto, F. Iikawa, R. Hey, and P. V. Santos, Phys. Rev. B 84, 205332 (2011).

${ }^{22}$ I. Kukushkin and V. Timofeev, Adv. Phys. 45, 147 (1996).

${ }^{23}$ A. Pinczuk, J. Shah, L. Stormer, R. C. Miller, A. C. Gossard, and W. Wiegmann, Surf. Sci. 142, 492 (1984).

${ }^{24}$ A. L. Efros, F. G. Pikus, and V. G. Burnett, Phys. Rev. B 47, 2233 (1993).

${ }^{25}$ R. J. Nicholas, R. J. Haug, K. v. Klitzing, and G. Weimann, Phys. Rev. B 37, 1294 (1988).

${ }^{26}$ S. L. Sondhi, A. Karlhede, S. A. Kivelson, and E. H. Rezayi, Phys. Rev. B 47, 16419 (1993).

${ }^{27}$ I. Mihalek and H. A. Fertig, Phys. Rev. B 62, 13573 (2000).

${ }^{28}$ V. S. Khrapai, A. A. Shashkin, E. L. Shangina, V. Pellegrini, F. Beltram, G. Biasiol, and L. Sorba, Phys. Rev. B 72, 035344 (2005).

${ }^{29}$ G. C. Kerridge, A. S. Plaut, M. C. Holland, C. R. Stanley, and K. Ploog, Phys. Rev. B 60, R5141 (1999).

${ }^{30}$ L. Gravier, M. Potemski, P. Hawrylak, and B. Etienne, Phys. Rev. Lett. 80, 3344 (1998). 